case of the Scottish data that revealed $77 \%$ of syphilis cases there to be type $14 \mathrm{~d}$.

\section{3-S1.03 PERFORMANCE OF REVERSE SEQUENCE SYPHILIS SCREENING IN JAMAICA}

doi:10.1136/sextrans-2011-050109.105

${ }^{1} \mathrm{M}$ Hobbs, ${ }^{2} \mathrm{~F}$ Gordon, ${ }^{2} \mathrm{C} \mathrm{J}$ Cooper, ${ }^{3} \mathrm{~S}$ Eastman, ${ }^{3} \mathrm{~T}$ Hylton-Kong, ${ }^{1} \mathrm{~S}$ Watson-Grant, ${ }^{1} \mathrm{~S}$ Weir, ${ }^{4} \mathrm{~J}$ P Figueroa. ${ }^{1}$ University of North Carolina, Chapel Hill, USA; ${ }^{2}$ Epidemiology Research and Training, Unit of the Ministry of Health, Jamaica; ${ }^{3}$ Comprehensive Health Centre, Center of Excellence, Jamaica; ${ }^{4}$ University of the West Indies, Jamaica

Background Algorithms for syphilis serologic testing traditionally have relied on screening with a non-treponemal test, such as the rapid plasma reagin (RPR) test or the toluidine red unheated serum test (TRUST) followed by confirmation using a treponemal test, such as Treponema pallidum particle agglutination (TP-PA). To reduce time, material and labour costs, many laboratories, including at the Comprehensive Health Centre in Kingston, Jamaica, have reversed the sequence testing first with a rapid treponemal test followed by non-treponemal testing of reactive sera.

Methods In a survey of STIs among men who have sex with men (MSM) in Jamaica, syphilis serologic testing is currently conducted using an initial rapid treponemal SD Bioline Syphilis 3.0 test followed by TRUST for reactive sera. Discordant sera that are Bioline-positive and TRUST-negative, or sera with TRUST titres $<$ or $=8$ undergo supplemental testing by TP-PA. SD Bioline was previously validated in the field and reference laboratory in Jamaica and is $95.2 \%$ sensitive and $93.5 \%$ specific compared to TP-PA. Here we report the results from sera obtained from 135 MSM in Kingston between December 2010 and February 2011.

Results Among 135 sera evaluated using the reverse syphilis screening sequence, 13 (9.6\%) had a positive rapid treponemal test. Among these 13 reactive sera, 6 (46.2\%) were nonreactive with TRUST. All discordant sera were also reactive by TP-PA, indicating that initial rapid testing did not produce false-positives in this setting. The proportion of discordant syphilis test results was similar among HIV+ and HIV- men. The prevalence of primary syphilis detected by concordant positive treponemal and nontreponemal tests in this survey was $5.2 \%$, compared to $5.3 \%$ in a previous survey conducted in this population during 2007-2008 using the traditional screening sequence.

Conclusions The prevalence of primary syphilis among MSM in Kingston has not changed since the previous survey. In the current survey using the reverse screening sequence, nearly half of sera that were reactive with the treponemal test produced discordant results with the non-treponemal test. Such results are consistent with previous syphilis infection, treated or untreated, or early primary syphilis in which non-treponemal antibodies have yet to develop. Distinguishing these possibilities requires detailed history and clinical assessment in addition to serologic test results.

\section{3-S1.04 PERFORMANCE CHARACTERISTICS OF BIOPLEX 2200 SYPHILIS IGG AND LIAISON TREPONEMA AUTOMATED ASSAYS FOR DETECTION OF ANTIBODIES TO TREPONEMA PALLIDUM}

doi:10.1136/sextrans-2011-050109.106

Y Fakile, S Kikkert, R Ballard, D Cox. Centers for Disease Control \& Prevention, Atlanta, USA

Background Serological testing continues to be a crucial tool for syphilis diagnosis and control. The commonly accepted syphilis screening algorithm is screening with non-treponemal tests such as RPR or VDRL, and confirming with treponemal tests such as TP-PA.
Recently, automation has been introduced whereby serological screening using treponemal tests has resulted in reduced labour time and removal of the subjectivity associated with the traditional testing algorithm. The objective of this study was to compare the performance characteristics of two FDA approved automated tests, the BioRad BioPlex 2200 Syphilis IgG and the DiaSorin LIAISON treponemal assays, with known predicate tests. The BioPlex 2200 syphilis IgG is a multiplex test that utilises three analytes (15-, 17-, \& 47-kDa) to detect specific IgG antibodies, whereas the LIASION treponemal assay uses only one analyte $(17 \mathrm{kDa})$ in a single step sandwich method to detect both syphilis IgG and IgM antibodies.

Methods A total of 1086 commercially obtained sera tested in this study consisted of: 430 from pregnant women, 409 from HIV positive individuals, and 111 from known syphilis patients of various disease stages. Characterised syphilis samples $(n=140)$ were also obtained from the CDC serum repository. All samples were screened by the Bioplex IgG, Liaison, RPR and TP-PA tests. Any indeterminate results were repeated at least once.

Results Of the 1086 samples tested, the syphilis reactivities were the following: $551(50.7 \%)$ by BioPlex IgG, 528 (48.6\%) by LIAISON, and $509(46.9 \%)$ by TP-PA. The sensitivity and specificity when compared to TP-PA for LIASION was $98.8 \%$ and $90.5 \%$ respectively. The BioPlex IgG sensitivity and specificity when compared to TP-PA was $85.1 \%$ and $80 \%$ respectively. Overall, $443(40.8 \%)$ samples were found to be reactive and $450(41.4 \%)$ non reactive to both LIAISON and BioPlex IgG. All three tests agreed on 877 (81\%) samples. On the 209 discordant samples TP-PA agreed with LIAISON 85.2\% $(n=178)$, BioPlex $7.2 \%(n=15)$, but disagreed with both tests $7.7 \%(n=16)$.

Conclusion Both tests have high throughput, walk-away capability, and would be useful in low prevalence settings. There was good agreement between the LIAISON and the BioPlex IgG in $893(82 \%)$ samples (Cohen's $\kappa=0.64$ ). The LIAISON had higher sensitivity most likely due to its detection of both IgG and IgM, while the BioPlex detected only IgG antibodies. Both tests show significant promise in the future of syphilis serology.

\section{3-S1.05 QUALITY ASSURANCE OF SYPHILIS TESTING IN A RURAL HEALTH FACILITY USING DRIED TUBE SPECIMENS (DTS)}

doi:10.1136/sextrans-2011-050109.107

${ }^{1} Y$ Adu-Sarkodie, ${ }^{1} A$ Dompreh, ${ }^{1} B$ Kofi Opoku, ${ }^{2} A$ Dzokoto, ${ }^{2} D$ Mabey, ${ }^{2} \mathrm{~B}$ Peeling. ${ }^{1}$ School of Medical Sciences KUMASI, Ghana; ${ }^{2}$ London School of Hygiene and Tropical Medicine, London, UK

Background Assuring the quality of a diagnostic test is important in healthcare. For syphilis testing, this includes proficiency testing of previously well characterised serum samples by health workers blinded to the results of characterisation. As part of a study on the feasibility of using a Point of Care (POC) rapid test devise for syphilis testing in rural antenatal settings, proficiency testing material prepared in a referral laboratory using Dried Tube Specimens (DTS) was sent for testing by the nurses/midwifes in these rural settings.

Methods Five well characterised DTS (one high RPR reactive, three low RPR reactives and one RPR non-reactive) were sent for on-site testing in eight rural antenatal facilities in Eastern Ghana. Training of nurses/midwifes in reconstituting the DTS and their testing and reporting was previously carried out. An instruction leaflet was enclosed in each batch of the DTS. Four rounds of the testing were carried out at monthly intervals.

Results Seven out of the eight facilities correctly reported results of the DTS for all the rounds. One facility however reported all specimens as negative at the first round. On-site investigation showed that the nurse running the antenatal clinic who normally wore reading glasses had lost them. On replacing her glasses and going through an on-site re-training, she obtained $100 \%$ in subsequent rounds of testing. 